\title{
Percutaneous nephrolithotomy tract dilatation techniques comparison (Balloon vs. Amplatz): Our Experience in Middle East.
}

\section{Adnan A. Abu-Qamar}

Jordanian Royal Medical Services

Mohanad M. Al-Naser

Jordanian Royal Medical Services

Ashraf S. Al-Majali ( $\triangle$ ALMAJALIASHRAF@YAHOO.COM )

Jordanian Royal Medical Services https://orcid.org/0000-0003-2653-2534

Mohammad A. Al-serhan MD4

Jordanian Royal Medical Services

Firas A. Al-Hammouri

Jordanian Royal Medical Services

Awad B. Al-kaabneh MDV

Jordanian Royal Medical Services

Samer G. Al-jfout MDQ

Jordanian Royal Medical Services

Ayman A. Al-Qaralleh

Jordanian Royal Medical Services

Mohammad A. Abdeldayem

Jordanian Royal Medical Services

Ahmad M. Al-Hiari

Jordanian Royal Medical Services

Laith F. Al-Khasawneh

Jordanian Royal Medical Services

Abdullah M. Al-rababaah MD12

Jordanian Royal Medical Services

Abdelhakim S. Ni'mate

Jordanian Royal Medical Services

\section{Belal A. Al-Khawaldah}

Jordanian Royal Medical Services

Monther A. Al-Emoush

Jordanian Royal Medical Services

Anees A. Hjazeen 
Jordanian Royal Medical Services

\section{Research article}

Keywords: Percutaneous Nephrolithotomy, Balloon dilatation, Amplatz dilatation, tract dilatation, nephrostomy

Posted Date: June 8th, 2020

DOI: https://doi.org/10.21203/rs.3.rs-33017/v1

License: (c) (1) This work is licensed under a Creative Commons Attribution 4.0 International License. Read Full License 


\section{Abstract}

Background: percutaneous nephrolithotomy (PCNL) is a gold widespread minimally invasive technique for the remedy of huge renal stones $>2 \mathrm{~cm}$. Our intention is to review the versions among the Balloon and Amplatz dilatation of percutaneous nephrolithotomy (PCNL) tract exploitation peri- and postoperative variables and make clear which technique is efficient.

Methods: 600 sufferers who underwent PCNLs among (1-April 2012 and 20-September 2018) in our Urology Center had been listed all through this retrospective look at, and divided into 2 groups: (group1) balloon tract dilatation (300 sufferers) and (group2) Amplatz serial fascial (ASF) dilatation (300 sufferers). Therefore, the demographic and categorical statistics were compared between each team.

Statistical evaluation used: SPSS software version twenty-four, T-test.

Results: operative time (balloon vs. Amplatz group: ( $85 \pm 32$ vs. $86.5 \pm 31$ ) mins; $P=$ zero.073), preoperative Hematocrit value ( $41.5 \pm 8.2$ vs. 40 .three $\pm 9.4 ; P=0.088)$, postoperative Hematocrit cost (36.1 \pm 2.2 vs. $34.6 \pm$ three. $4 ; P=0.023)$, blood transfusion levels $(10.6 \%$ vs. $12.1 \% ; P=0.002)$, tract dilatation trials $(20.4 \%$ vs. $22.6 \% ; P=0.051)$, stone clearance price (78.three\% vs. $77.8 \% ; P=0.083)$, and therefore, the versions regarding the price have been higher slightly in balloon cluster while within the opportunity variables had been statistically insignificant besides blood transfusion rate and postoperative hospital live.

Conclusion: The balloon dilatation is price effective than amplatz method in manner of postoperative hospital live length and blood transfusion, whereas most of the other diverse factors like safety, operative period, achievement rate, and stone clearance price; they gave comparable consequences.

\section{Background}

Management of urinary stones by using open surgical procedure has been replaced by minimally invasive processes, like percutaneous nephrolithotomy (PCNL), retrograde intrarenal surgical treatment (RIRS), extracorporeal shock wave lithotripsy (ESWL), laparoscopy procedures, and aggregate of these techniques ${ }^{1}$. Percutaneous nephrolithotomy (PCNL) has been notion-about as a 'gold trendy' minimally invasive surgical modality for massive renal calculi $>2 \mathrm{~cm}$, lower calyceal stones sized ten to twenty millimeter with unfavorable elements or refractory to shockwave therapy (ESWL) considering Fernstrom and Johansson first removed kidney stones thru a nephrostomy tract in $1976^{2}$. There are (5) ranges of this operation: (1)coming into to the collecting gadget and making an get entry to (guided by using sonography or fluoroscopy), (2)tract dilatation by means of 3 well-known strategies: semi-inflexible Amplatz dilatation, Alken kind metallic telescopic dilatation, and balloon dilatation, (3)placement of the sheath, (4)disintegration of the stones, and (5)placement of the nephrostomy tube ${ }^{3}$. With regards to obtaining access to the collecting system by using radiology, there are two number one methods: the triangulation technique (TT) and so the "eye of the needle" (EN) method ${ }^{4}$. There are diverse warning 
signs for percutaneous nephrostomy apart from lithotripsy: 1- alleviation of urinary obstruction specially as soon as first-line endourological approaches failing, 2- urinary diversion for varies functions, and threefor diagnostic testing. Thus, it's essential to be conversant in most of the nephrostomy getting get admission to and tract dilatation strategies to decrease the headaches, the operative time, and blood loss of this minimally invasive system ${ }^{5}$. In addition to the form of tract dilatation technique that we'll talk for the duration of this observe there, rectangular measures more than one risk elements account for the complications drastically haemorrhage post PCNL: stone size, staghorn stone, operation time, and diploma of hydronephrosis ${ }^{6}$. Many studies created a contrast between completely different strategies of tract dilatation in $\mathrm{PCNL}^{7}$. A range of them showed the superiority of balloon dilatation technique over the amplatz method in affected person outcomes ${ }^{8}$. Whereas the style of the others demonstrates that the amplatz methodology is safer and much less complex than metallic telescopic dilation PCNL ${ }^{9}$. On the alternative hand; several studies suggested the ascendency of amplatz PCNL over balloon PCNL ${ }^{10}$. In our retrospective take a look at, we tend to theorize that preoperative and postoperative effects of every strategy (amplatz and balloon dilatation) square degree similar besides inside the price facet, that plenty of in balloon technique.

\section{Methods}

This retrospective look at turned into finished within our urology institute and enclosed regarding (six hundred) patients who underwent PCNLs via either one among the 2 strategies of tract dilatation (Amplatz and balloon dilation) between (1-April 2012 and 20-September 2018) as an elective management for removing huge renal calculi $>2 \mathrm{~cm}$, decrease group calyceal stones sized ten to twenty millimeter with unfavorable elements or refractory to ESWL were definitive treatment changed into the case. The patients had been the applicants for either technique in line with the treating urologist and interventional radiotherapist desire as each approach square degree picks of PCNL tract advent.

The inclusion criteria have been aged higher than the pediatric cohort, symptomatic renal stones without or with hydronephrosis, large stones $>2 \mathrm{~cm}$, lower group stones sized ten to 20 millimeter with unfavorable factors or refractory to ESWL, nonobstructed kidney without signs and symptoms of infection and every body have been the candidates for either method. Whereas, the exclusion criteria were pregnant, uncontrolled excessive blood pressure or coagulopathy, ureteric obstruction, single or transplanted kidney, history of latest dialysis, urine bladder and renal tumors. Once getting the scientific facts of these sufferers, a revision of those documents had been fashioned looking at the history of the past medical sicknesses, the chief complaints, the records of the offering illnesses, the bodily examinations, and every one investigation became accomplished for those patients like (complete blood count, kidney feature tests, electrolytes, urine evaluation, and cultures, radiology imagings along with (simple stomach $\mathrm{x}$-rays, renal sonography, and renal computed tomography)). Then our evaluation assistant divided the patients into a pair of groups in keeping with the tract dilatation approach turned into used as follows: organization one (300) patients who undergone PCNL through balloon tract dilatation technique, institution two contains (300) sufferers who underwent amplatz dilation PCNL. The 
age bracket of all sufferers become went among (18 and 65 years), with a mean value of (forty three years) and consequently, the same old deviation of $(S D=12.958)$. Therefore, the age teams have been between (19 and 64 years) for group one, and among (18-65 years) for group two, whereas the mean fee, and therefore, the standard deviation (SD) of the age teams had been (44.seventy five years, $S D=11.95$ ) and (41.18 years, $S D=13.39)$ for the organization one, and group, respectively.

All patients got second or third-technology cephalosporin earlier than and in any case PCNLs until finishing off a full direction for every antibiotic, in step with the patient clinical state of affairs and with the adjustment of each antibiotic dose if there was bobbing up within the kidney characteristic tests. The length of and everyone complication of the procedures that were done by using the 2 strategies were recorded inside the consequences below, taking into the idea that the follow-up duration of the headaches changed into beginning from throughout tract dilatation to three months. Operative time started out from the cystoscopic examination up to the nephrostomy tube insertion with suturing to the skin. The hospitalization period turned into from the operative-day until the discharging day. Blood chemistries and urine subculture tests have been taken preoperatively. Patients, whose urine cultures were determined to be positive, have been given appropriate antibiotics and underwent surgical procedures as soon as terrible cultures were acquired. The full-size fall in Hematocrit levels have been estimated in the idea of pre-, and postoperative hemoglobin values, and consequently, the full quantity of blood transfusion.

PCNL technique: after anesthesia, whereas, the affected person turned into positioned inside the supine design, the set become changed to the lithotomy role, and thru a twenty-two F cystoscope; transurethral cystoscopy was executed and an open-ended $6 \mathrm{~F}$ ureteral tube turned into inserted as much as the kidney that containing the stone. Then the patients were put within the prone role for the coming into the intrarenal collecting machine under the fluoroscopy steering. A radio-opaque contrast turned into given thru a ureteral catheter for a retrograde pyelogram. Eighteen Gauge $(\mathrm{G})$ percutaneous get admission to needle changed into inserted into the pelvicalyceal system through the calyx from which the most of stones may be extracted with the lowest bleeding risk. Once urine outflow turned into ascertained by discharging from this needle, the guidewire was inserted in the needle until achieving the pelvicalyceal gadget. After incising the pores and skin with a twenty $\mathrm{G}$ surgical knife, $6 \mathrm{~F}$, then ten $\mathrm{F}$ co-axial dilators have been used to dilate the percutaneous nephrostomy tract over the guidewire. Over the guidewire, Balloon dilator (UltraxxTM nephrostomy balloon catheter, Cook Group, USA) become advanced into the focused calyx, then inflation of this balloon up to fifteen $\mathrm{kg} / \mathrm{cm} 2$ atmospheric strain was carried out using the radio-opaque agent and with the assist of an inflator (Nephrostomy inflator device, Cook). After these steps, a thirty $\mathrm{F}$ working sheath changed into advanced into the pelvicalyceal machine over the balloon and then withdrawn of this balloon after deflation changed into befell. On the other side, the tract dilation by Amplatz dilators changed into passed off by means of the following steps: over the guidewire, the percutaneous tract turned into increased up to 20-eight $F$ through these dilators, then the targeted calyx and consequently the pelvicalyceal machine become entered with the aid of thirty F Amplatz sheath (Amplatz renal dilator Set, Cook Group, USA) over those co-axial dilators. 
The stones disintegration and clearance: using a twenty- $5 \mathrm{~F}$ nephroscope, targeted stones have been disintegrated through pneumatic or ultrasonic lithotripter with the help of the forceps. After entire stone clearance, simply one entry changed into executed. Throughout operation in the beginning chance, the retrograde ureteral catheter turned into stored in, and the guidewire was withdrawn after insertion of a Foley catheter size $16 \mathrm{~F}$ into the pelvis as a nephrotomy tube. After termination of the operation and fixation of the nephrostomy tube, compression and dressing of the operative site turned into carried out, then repositioning the affected person to the supine set for transferring him to the restoration ward.

While in the ward, if hemorrhage turned into mentioned thru nephrostomy tube, then the tubes had been clamped. If the coloration of the urine became clear, then the Foley catheter and retrograde angiocatheter had been withdrawn within the future morning. On put up-procedural one and a couple of Days, the selection of clamping and withdrawn of the nephrostomy tubes turned into taken if the patients have been no longer febrile, no residual stones were seen, and they were now not having a distressing pain. A retrograde nephrostogram became acquired if there's a suspicion of urinary leak or residual obstructive ureteral stones. Then if loose passage of the radio-opaque comparison into the bladder changed into ascertained, then nephrostomy tubes had been taken out. While if there's a suspicion of urine leak or residual stones that handed and impacted inside the ureter during the postoperative duration, then Double-J ureteral stents were deep-seated in these patients. The patients had been evaluated with renal CT stone protocol at three months of the postoperative period. The operation turned into deemed to achieve achievement if the stone-unfastened state changed into done or the nonobstructive, symptomless, noninfectious, clinically insignificant residual fragments (CIRFs) but four millimeters were decided postoperatively inside the tract.

Most of the facts become presented within the fashion of tabulated comparative statistics, the numbers and consequently, the odds had been generated from the categorical statistics with the aid of the usage of SPSS software model (24), and consequently, the Comparison between this categorical data ( $\mathrm{N}(\%)$ ) changed into achieved by means of T-test. P-value $<0.05$ become idea of as statistically good sized.

Ethical committee approval become gained from our Royal Medical Services Ethical committee institution for book of this take a look at.

\section{Results}

Regarding the age groups that we tend tore mentioned inside the strategies we calculated the chances and SD and vicinity them in demographic characteristics desk (table 1), beside the range, and therefore, the mean price of the stone size \pm SD for all patients $(1-6.7 \mathrm{~cm}, 3$. fifty three $\mathrm{cm} \pm 1.63 \mathrm{~cm}$, respectively) and for each group (group1: $1.2-6.7 \mathrm{~cm}$, three. $48 \mathrm{~cm} \pm 1.6 \mathrm{~cm}$, respectively), (institution 2: 1-6.three $\mathrm{cm}$, $3.35 \mathrm{~cm} \pm 1.57 \mathrm{~cm}$, respectively).

We tend to observe there rectangular degree more than one variations among balloon (organization one) and Amplatz tract (institution ) dilatation techniques of PCNLs according to: 1-operative time: mean price \pm sd for institution 1 and organization 2 ( $85 \pm 32$ minutes vs. $86.5 \pm 31$ minutes, respectively; $p=0.073$ ), 2- 
Hematocrit degree preoperative: mean price $\pm s d(41.5 \pm 8.2$ vs. 40 .three \pm 9.4 , respectively; $p=0.088)$ and post-operative ( $36.1 \pm 2.2$ vs. $34.6 \pm 3.4$, respectively; $p=0.023)$, three- blood transfusion rates for the procedures had been carried out through each technique: mean price $(10.6 \%$ vs. $12.1 \%$, respectively; $p=$ 0.002), 4- tract dilatation failure (which changed into dealt by using different further system for stone elimination like ESWL, RIRS, laparoscopy, an opportunity preference or trial of tract dilatation, and even open surgical procedure): mean value ( $20.4 \%$ vs. $22.6 \%$, respectively; $p=0.051)$ or achievement charge: mean cost $(79.6 \%$ vs. $77.4 \%$, respectively; $p=0.062), 5$ - stone clearance rate: mean cost $(78.3 \%$ vs. $77.8 \%$, respectively; $p=0.083), 6$ - hospital stay for all sufferers with mean value \pm popular deviation (3.forty three \pm 1.76 ) and for group one (balloon technique) vs. organization (amplatz approach): (three. $21 \pm 2.94 \mathrm{vs.}$ $2.72 \pm 1.85$, respectively; $p=0.027$ ) and ultimately 7 - the fee of every method (which was barely higher in balloon method), (Table 2).

\section{Table 1: Including the demographic characteristics of each team.}

\begin{tabular}{lllll} 
& All patients & Group 1 & Group 2 & P value \\
\hline Pts. Ages (mean \pm SD) years & $(43 \pm 12.96)$ & $(44.75 \pm 11.95)$ & $(41.18 \pm 13.39)$ & 0.054 \\
\hline Stone sizes (mean \pm SD) cm & $(3.53 \pm 1.63)$ & $(3.48 \pm 1.6)$ & $(3.35 \pm 1.57)$ & 0.097
\end{tabular}

P-value was estimated between group one and group two.

Table 2: comparisons between each group concerning the various variables.

\begin{tabular}{llll} 
The study variables & Group $1\left(\mathrm{M}^{\star} \pm \mathrm{SD} \AA\right)$ & Group 2 $(\mathrm{M} \pm \mathrm{SD})$ & P value \\
\hline Operative time (minutes) & $(85 \pm 32)$ & $(86.5 \pm 31)$ & 0.073 \\
\hline Preoperative Hematocrit level $(\mathrm{gr} \backslash \mathrm{dl})$ & $(41.5 \pm 8.2)$ & $(40.3 \pm 9.4)$ & 0.088 \\
\hline Postoperative Hematocrit level $(\mathrm{gr} \backslash \mathrm{dl})$ & $(36.1 \pm 2.2)$ & $(34.6 \pm 3.4)$ & 0.023 \\
\hline Blood transfusion rates $(\%)$ & $(10.6)$ & $(12.1)$ & 0.002 \\
\hline Tract dilatation success rate $(\%)$ & $(79.6)$ & $(77.4)$ & 0.062 \\
\hline Tract dilatation failure $(\%)$ & $(20.4)$ & $(22.6)$ & 0.051 \\
\hline Stone clearance rate $(\%)$ & $(78.3)$ & $(77.8)$ & 0.083 \\
\hline Hospital stay (days) & $(3.21 \pm 2.94)$ & $(2.72 \pm 1.85)$ & 0.027 \\
\hline The cost of the technique & Slightly higher & Slightly lower &
\end{tabular}

$M^{*}=$ Mean. $S D \AA=$ Standard Deviation 


\section{Discussion}

According to our ends up in this examine, we have a tendency to note that the balloon dilatation technique for PCNL had an equivalent operative period time, a lower in the Hematocrit stage publish the system, success, and failure charge of tract dilation beside the stone clearance fee in comparison to amplatz method. Whereas the blood transfusion fee changed into lower in the balloon method organization, the hospital stays, and consequently, the fee of the method became barely higher. No versions have been observed regarding X-ray exposure between the 2 techniques on account that no ultrasound guidance became used and each technique were depending on continuous radiology exposure all through the dilatation of the PCNL tract. Also, tract dilatation by means of amplatz in our analysis because of the retrospective observe aspect turned into completed by way of serial dilatation, no longer via unmarried-shot dilatation method which provides shorter operative time and less radiation exposure in keeping with maximum of the literature $11,12,13$.

Some original research pronounced that the balloon approach isn't not so good as amplatz dilators regarding the hardship price, however, a rise of stone clearance and reduce the blood loss square degree dependent chiefly on urologist expertise ${ }^{14}$.

Ren Minghua said in an original article that the balloon method had a better tract get entry to fulfillment charge, shorter tract dilatation time, less blood loss, and decrease proportions of circulatory overload, and postoperative fever ${ }^{15}$.

On the opposite aspect, Hamid Pakmanesh et al. observed that bleeding and different headaches had been comparable inside the balloon and amplatz teams, whereas operative time become longer within the amplatz organization with a higher charge of short dilation. Concerning the approaching the decrease organization calyces, the balloon technique become superior ${ }^{16}$.

Concerning renal feature preservation inside the sufferers who underwent PCNL by different techniques of tract dilatation consisting of balloon vs. Amplatz, all strategies had comparable effects, which became documented by means of Ali Unsal and collages ${ }^{17}$.

Davidoff R. Et al. Supported our consequences regarding lower transfusion fee within the balloon group in comparison to the amplatz group inside the article entitled "Influence of approach of percutaneous tract creation at the occurrence of renal hemorrhage" ${ }^{18}$.

The fee of the balloon dilation group turned into more than an amplatz institution, that turned into reported by using Burak Oczift and collages in a study became posted through the Turkish magazine of urology 19 .

Kidney hypermobility was problematic at some point of Amplatz dilatation that became more feepowerful than balloon approach, however, a comparable concerning effectivity, speed, and protection, those outcomes had been stated in an article published on May 2008 by Journal of Endourology 20 . 
Limitations of our have a look at: thanks to the retrospective side of the study, and negative documentation gadget, we lack the calculated time for radiation exposure during the tactics that have an effect at the evaluation facet of X-ray exposure among each technique.

\section{Conclusion}

The balloon tract dilation approach in PCNL has the benefit of decreased blood transfusion charge than the amplatz method; however, it has a prolonged hospital stay and barely an additional charge than the latter. Whereas the techniques have similar effectivity, rapidity, and protection in reference to the achievement fee of tract dilation and stone clearance charge, operative time and postoperative trouble fee. So, the use of each approach is really appropriate for PCNL tactics without regulations once they may be available within the urology department consistent with the sufferers' suitable characteristics for those strategies.

\section{Abbreviations}

PCNL: Percutaneous nephrolithotomy.

ESWL: extra corporeal shock wave lithotripsy.

SD: standard deviation

DJC: double J catheter.

(CIRFs): clinically insignificant residual fragments.

(ASF): Amplatz serial fascial.

(TT) : triangulation technique

(EN): "eye of the needle"

\section{Declarations}

\section{Ethics approval and consent to participate:}

Ethical committee approval become gained from our Royal Medical Services Ethical committee institution for book of this take a look at. This study is retrospective and there was no need for patients consent.

\section{Consent for publication:}

Not Applicable. 


\section{Availability of data and materials:}

Data about manuscript is available when is needed at corresponding author email almajaliashraf@yahoo.com.

\section{Competing interests:}

"The authors declare that there is no conflict of interest regarding the publication of this paper."

\section{Funding:}

"This research received no external funding"

\section{Authors' contributions:}

"Conceptualization, $\mathrm{AAQ}$ and $\mathrm{FH}$; methodology and software, AN, SJ, AQ, MA and ANHJ; validation, BK and ME; formal analysis, AHHI, LK; investigation and resources, MS, AR; data curation, AK; writingoriginal draft preparation and writing-review and editing, AM; visualization, MN; supervision, AAQ; project administration and funding acquisition, AM. All authors have read and agreed to the published version of the manuscript."

\section{Acknowledgments}

"The authors declare that there is no Acknowledgments regarding the publication of this paper."

\section{References}

1- Huseyin Celik, Cemal Tasdemir, Ramazan Altintas, et al. AN OVERVIEW OF PERCUTANEOUS NEPHROLITHOTOMY:EMJ Urol. 2015;3:46-52.

2-Srirangam SJ, Darling R, Stopford M, Neilson D. Contemporary practice of percutaneous nephrolithotomy: review of practice in a single region of the UK. Ann R Coll Surg Engl. 2008;90(1):40-44.

3-Necmettin Penbegul, Onur Dede, Mansur Daggulli, Namik Kemal Hatipoglu, Yasar Bozkurt. A novel percutaneous nephrolithotomy (PCNL) set: The 'Economical One-shot PCNL Set' (Ecoset), Arab Journal of Urology, Volume 15, Issue 3, 2017, Pages 199-203.

4-Budak, Salih \& Yucel, Cem \& Kisa, Erdem \& Kozacioglu, Zafer. Comparison of two different renal access techniques in one-stage percutaneous nephrolithotomy: triangulation versus eye of the needle. Annals of Saudi medicine 2018; 38: 189-193.

5-Dagli M, Ramchandani P. Percutaneous nephrostomy: technical aspects and indications. Semin Intervent Radiol. 2011; 28(4): 424-437. 
6- Xiangjun Meng, Juan Bao, Qiwu Mi, and Shaowei Fang, "The Analysis of Risk Factors for Hemorrhage Associated with Minimally Invasive Percutaneous Nephrolithotomy," BioMed Research International, vol. 2019, Article ID 8619460, 6 pages, 2019.

7- Yamaguchi, Akito \& Skolarikos, Andreas \& Buchholz, Noor et al. Operating Times and Bleeding Complications in Percutaneous Nephrolithotomy: A Comparison of Tract Dilation Methods in 5537 Patients in the Clinical Research Office of the Endourological Society Percutaneous Nephrolithotomy Global Study. Journal of endourology / Endourological Society. (2011); 25: 933-939.

8- A. R. Kalpee, R. Venter, T. Fourie,ET AL. Single-centre comparison of a novel single step balloon inflation device and Amplatz sheath dilatation during percutaneous nephrolithotomy: A pilot study.S Afr J Surg 2012;50(3):79-81. DOI:10.7196/SAJS.1071.

9- Hijazi S, Echtle D, Hasselhof VM, Trojan L, Heinrich E. Metal telescopic and Amplatz sheath dilation in nephrolithotomy. Urol Ann. 2016;8(1):66-69. doi: 10.4103/0974-7796.163795.

10- Lopes, Tomé \& Sangam, Kandasami \& Alken, Peter et al. The Clinical Research Office of the Endourological Society Percutaneous Nephrolithotomy Global Study: Tract Dilation Comparisons in 5537 Patients. Journal of endourology / Endourological Society. (2011); 25: 755-62. Doi: 10.1089/end.2010.0488.

11- Nour HH, Kamal AM, Zayed AS, Refaat H, Badawy MH, El-Leithy TR. Single-step renal dilatation in percutaneous nephrolithotomy: A prospective randomised study. Arab J Urol. 2014;12(3):219-222. doi:10.1016/j.aju.2014.06.001.

12- P V, Thiruvarul \& P, Periasamy \& Balashanmugam K, Pitchai et al. SINGLE STEP VS SERIAL DILATATION FOR TRACT CREATION IN PERCUTANEOUS NEPHROLITHOTOMY: A RANDOMIZED CONTROLLED TRIAL. Journal of Evolution of Medical and Dental Sciences. (2015); 4: 15669-15672. Doi: $10.14260 /$ jemds/2015/2254.

13-X. Jing, S. Ying, Z. Xiaoping, ET AL. Chinese One-shot Dilation versus Sequential Fascial Dilation for Percutaneous Nephrolithotomy: A Feasibility Study and Comparison 2019;Vol. 16 No. 01: P:21-26.

14- Chen P-H, Yan M-Y, Chiang H-C, et al. Effect of Different Years of Resident Training on Complications and Stone Free Rate during Percutaneous Nephrolithotomy for Large Renal Stone Greater Than $20 \mathrm{~mm}$ : A Prospective Observational Study Using Balloon Dilator. Clin Surg. 2018; 3: 2098.

15- Ren Minghua, Zhang Cheng, Fu Weijun, et al. Balloon dilation versus Amplatz dilation during ultrasound-guided percutaneous nephrolithotomy for staghorn stones:Chin Med J 2014;127 (6): 10571061.

16- Hamid Pakmanesh, Azar Daneshpajooh, Mahboubeh Mirzaei, et al., "Amplatz versus Balloon for Tract Dilation in Ultrasonographically Guided Percutaneous Nephrolithotomy: A Randomized Clinical Trial," 
BioMed Research International, vol. 2019, Article ID 3428123, 8 pages, 2019.

17- Ali U“ nsal, Go"khan Koca, Berkan Res, et al.Effect of Percutaneous Nephrolithotomy and Tract Dilatation Methods on Renal Function: Assessment by Quantitative Single-Photon Emission Computed Tomography of Technetium-99m-Dimercaptosuccinic Acid Uptake by the Kidneys:JOURNAL OF ENDOUROLOGY 2010; Volume 24, Number 9: p(1-6).

18- Ramin Davidoff, Gary C. Bellman,et al. Influence of Technique of Percutaneous Tract Creation on Incidence of Renal Hemorrhage, The Journal of Urology, Volume 157, Issue 4, 1997, Pages 1229-1231.

19- Özçift B, Bal K, Dinçel Ç. A comparison of balloon and amplatz dilators in percutaneous nephrolithotomy: a retrospective evaluation. Turk J Urol. 2013;39(4):226-231. doi:10.5152/tud.2013.058.

20- Gonen, Murat \& Mustafa Istanbulluoglu, Okan \& Cicek, Tufan \& Ozturk, Bulent \& Ozkardes, Hakan. (2008). Balloon Dilatation versus Amplatz Dilatation for Nephrostomy Tract Dilatation. Journal of endourology / Endourological Society. 22. 901-4. 10.1089/end.2007.0167. 